\title{
Two-Dimensional Dynamic Localization of Light
}

\author{
Alexander Szameit ${ }^{1, *}$, Ivan L. Garanovich ${ }^{2}$, Matthias Heinrich ${ }^{3}$, Andrey A. Sukhorukov ${ }^{2}$, \\ Felix Dreisow ${ }^{3}$, Stefan Nolte ${ }^{3}$, Andreas Tünnermann ${ }^{3}$, Stefano Longhi ${ }^{4}$, and Yuri S. Kivshar ${ }^{2}$ \\ ${ }^{1}$ Solid State Institute and Physics Department, Technion, 32000 Haifa, Israel \\ ${ }^{2}$ Nonlinear Physics Centre and Centre for Ultra-high bandwidth Devices for Optical Systems (CUDOS), \\ Research School of Physics and Engineering, Australian National University, Canberra, ACT 0200, Australia \\ ${ }^{3}$ Institute of Applied Physics, Friedrich-Schiller-University Jena, Max-Wien-Platz 1, 07743 Jena, Germany \\ ${ }^{4}$ Dipartimento di Fisica and Istituto di Fotonica e Nanotecnologie del CNR, \\ Politecnico di Milano, Piazza L. da Vinci 32, 20133 Milano, Italy \\ *Phone +972 (0)4829 2183; Fax +972 (0)4823 5107; Email: szameit@techunix.technion.ac.il
}

We report on the first experimental observation of two-dimensional dynamic localization of light. We demonstrate suppression of beam diffraction in femtosecond laser-written modulated waveguide arrays of hexagonal and zig-zag geometries.

\section{(C) 2009 Optical Society of America}

OCIS codes: (130.3120) Integrated optics devices; (350.3390) Laser materials processing

Similarities between wave optics and wave mechanics led to the discovery of various analogies between quantum and optical phenomena over the past two decades [1]. It was shown that the temporal evolution of the electronic wave function is similar to the spatial light propagation along guiding dielectric structures. Based on this analogy, many effects predicted originally in the context of solid-state physics were first observed in optics [1]. The main advantage of such waveguide systems is that in optics the evolution takes place in space instead of time, allowing the direct observation by monitoring the spatial light propagation [2].

Importantly, optics provides a platform which goes beyond pure mimicking of quantum mechanics; it rather allows to analyze systems which are much more sophisticated than common settings in solid state physics. One important example is Dynamic Localization (DL), the suppression of broadening of a particle wave packet during its motion in a periodic potential under the action of externally applied ac electric field [3], a setting which is fundamentally distinct from Bloch oscillations under an invariant dc potential. The same effect can occur for optical wave packets in curved waveguide arrays, where the waveguide curvature [Fig. 1(b)] has the role of the driving field, in this case canceling diffraction [4]. It was predicated that DL occurs in multidimensional lattices at a series of resonances between lattice, particle and driving-field parameters $[3,5]$. However, only partial DL resonances associated with some of the lattice directions were observed in two-dimensional systems up to now [6].

Here, we report for the first time on suppression of diffraction broadening of a two-dimensional wave packet in hexagonal and zig-zag shaped arrays of periodically curved optical waveguides, where light is forced to exhibit periodic oscillations in width as a result of the two-dimensional DL effect. We employ the laser direct-writing method [7] to inscribe one- and two-dimensional waveguide arrays with required bending profiles [Fig. 1(b)] in fused-silica glass.

First, we study DL of light beams in hexagonal photonic lattices, where the transverse dynamics of a wave packet occurs by evanescent coupling between the individual lattices sites [Fig. 1(a)] and is described by the coupled mode equations [5],

$$
i \frac{d \Psi_{n, m}}{d z}+C_{1}^{*} \Psi_{n-1, m}+C_{1} \Psi_{n+1, m}+C_{2}^{*} \Psi_{n, m-1}+C_{2} \Psi_{n, m+1}+C_{3}^{*} \Psi_{n-1, m+1}+C_{3} \Psi_{n+1, m-1}=0,
$$

where $\Psi(z)_{n, m}$ is the propagating field amplitudes in the $n, m$ th lattice site, $C_{1}=C \exp \left[-i \omega \dot{x}_{0}(z)\right]$ and $C_{2}=C_{3}=$ $C \exp \left[-i \omega \dot{x}_{0}(z) / 2\right]$ are the coupling coefficients between the neighboring waveguides [Fig. 1(a)]. Here dot stands for the derivative along $z, \omega=2 \pi n_{0} d / \lambda$ is the dimensionless frequency, $\lambda$ is the wavelength, $n_{0}$ is the average refractive
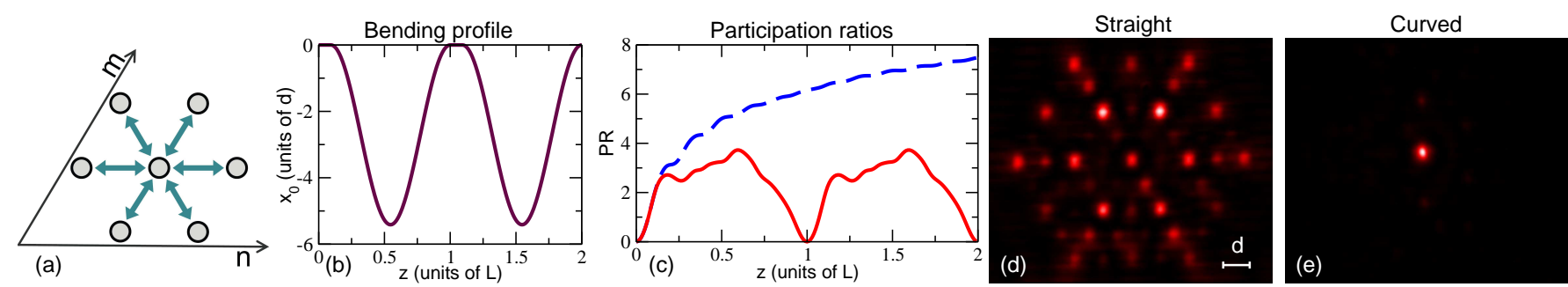

Fig. 1. Dynamic localization of light in hexagonal photonic lattice. (a) Sketch of the couplings in the hexagonal lattice. (b) Periodic waveguide bending profile. (c) Numerically-computed participation ratios for the straight (dashed) and curved (solid) lattices. (d,e) Experimentally measured output beam profiles for the straight and curved lattices. 


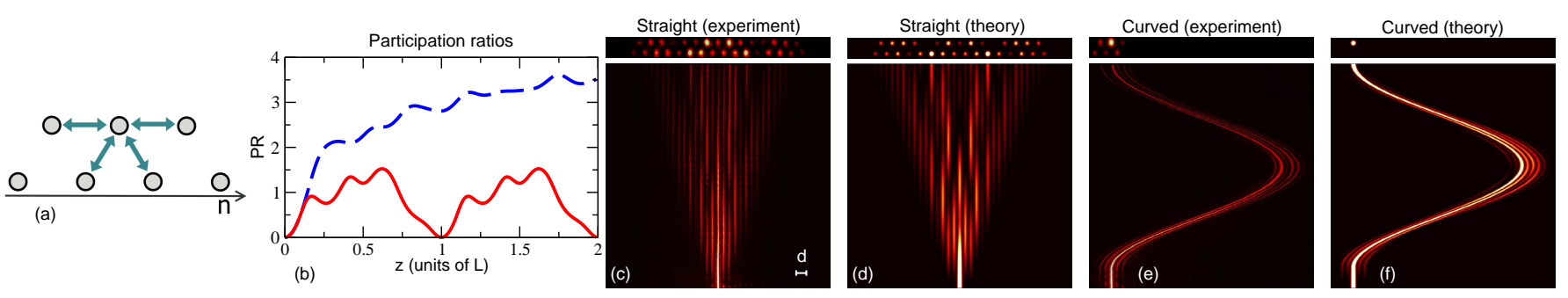

Fig. 2. Dynamic localization of light in zig-zag waveguide array. (a) Sketch of the couplings in the zig-zag array. (b) Calculated numerically participation ratios for the straight (dashed) and curved (solid) arrays. (c) Experimentally measured output beam profiles (top) and fluorescent images of beam propagation (bottom) for the straight array. (d) Numerically-computed output beam profiles (top) and beam propagation (bottom) for the straight array. (e,f) The same as (c) and (d) for the curved array.

index, $d$ is the waveguide spacing, and asterisk stands for the complex conjugation. The real-valued coefficient $C$ defines the coupling strength, and it directly characterizes diffraction rate in a straight hexagonal waveguide array with $x_{0} \equiv 0$ [see Fig. 1(d)]. In our setting the waveguides are periodically bent in the $(x-z)$-plane along the propagation direction $z$, and this is expressed mathematically through the transverse shift of waveguides $x_{0}(z)$.

We have previously predicted [5] that two-dimensional DL regime associated with complete suppression of beam broadening is realized with a bending profile which consists of alternating straight and sinusoidal segments in each period $L$,

$$
x_{0}(z)=\left\{\begin{array}{r}
0, \text { if } 0 \leq z \leq z_{0} \\
A\left\{\cos \left[2 \pi\left(z-z_{0}\right) /\left(L-z_{0}\right)\right]-1\right\}, \text { if } z_{0} \leq z \leq L
\end{array} .\right.
$$

where $z_{0}=\left[1-1 / \mathrm{J}_{0}(\xi)\right]^{-1} L, A=-z_{0} \xi / \pi \omega \mathrm{J}_{0}(\xi), \mathrm{J}_{0}$ is the Bessel function of the first kind of zero order, and $\xi \simeq 2.61$ is determined from the equation $\mathrm{J}_{0}(\xi)=\mathrm{J}_{0}(2 \xi)$. The resulting bending profile for our $L=25 \mathrm{~mm}$ long samples with the intersite spacing of $d=22 \mu \mathrm{m}$ is shown in Fig. 1(b). We characterize the rate of the beam diffraction broadening by the participation ratio defined as $P R=\ln \left[\left(\sum_{\mathrm{n}}\left|\Psi_{n}\right|^{2}\right)^{2} / \sum_{\mathrm{n}}\left|\Psi_{n}\right|^{4}\right]$. In Fig. 1(c) the numerically-computed participation ratios for infinite hexagonal lattices are shown: in the curved lattice beam experiences periodic refocusing in the DL regime (solid curve) in contrast to the diffraction broadening in the straight array (dashed curve). Experimental output intensity distributions obtained for our finite-size samples (hexagon-shaped boundaries with 5 waveguides at each facet) when the central waveguide is excited at the input facet with $\mathrm{cw}$ laser light at $\lambda=633 \mathrm{~nm}$ are shown in Figs. 1(d) and 1(e). One clearly sees the full suppression of the beam diffraction in all transverse directions in the curved sample, in sharp contrast to the strong diffraction in the straight sample.

Interestingly, a similar DL phenomenon can also be observed in zig-zag lattices which are described by the Eq. (1) with $m=1,2$ [see Figs. 2(a)]. Participation ratios calculated for infinite zig-zag arrays with bending period and waveguide spacing matched to the experimental values $L=100 \mathrm{~mm}$ and $d=26 \mu \mathrm{m}$ are shown in Figs. 2(b), where one can observe beam DL in the curved array (solid curve) and diffraction broadening in the straight array (dashed curve). To visualize the intensity distribution inside the lattice, we used a fluorescence microscopy technique [2]. Our samples contain 16 waveguides in the bottom row $(m=1)$ and 15 waveguides in the top row $(m=2)$, the beam is launched in the central waveguide in the top row. Recorded fluorescence images, showing discrete diffraction along the straight and curved arrays, are shown in Figs. 2(c) and 2(e) and compared with theoretical predictions [Figs. 2(d) and 2(f)]. Note the excellent agreement between experimental results and the theoretical predictions.

In conclusion, we presented the first experimental demonstration of two-dimensional dynamic localization effects using hexagonal and zig-zag shaped curved photonic lattices. Our results open up new opportunities for diffraction control and flexible shaping of light beams in photonic lattices.

\section{References}

1. S. Longhi, "Quantum-optical analogies using photonic structures," Laser Photon. Rev. 3, $243-261$ (2009).

2. A. Szameit, F. Dreisow, H. Hartung, S. Nolte, A. Tünnermann, and F. Lederer, "Quasi-incoherent propagation in waveguide arrays," Appl. Phys. Lett. 90, 241113-3 (2007).

3. D. H. Dunlap and V. M. Kenkre, "Dynamic localization of a charged particle moving under the influence of an electric field," Phys. Rev. B 34, 3625-3633 (1986).

4. S. Longhi, M. Marangoni, M. Lobino, R. Ramponi, P. Laporta, E. Cianci, and V. Foglietti, "Observation of dynamic localization in periodically curved waveguide arrays," Phys. Rev. Lett. 96, 243901-4 (2006).

5. I. L. Garanovich, A. Szameit, A. A. Sukhorukov, T. Pertsch, W. Krolikowski, S. Nolte, D. Neshev, A. Tünnermann, and Yu. S. Kivshar, "Diffraction control in periodically curved two-dimensional waveguide arrays," Opt. Express 15, 9737-9747 (2007).

6. A. Szameit, I. L. Garanovich, M. Heinrich, A. A. Sukhorukov, F. Dreisow, T. Pertsch, S. Nolte, A. Tünnermann, and Yu. S. Kivshar, "Polychromatic dynamic localization in curved photonic lattices," Nature Phys. 5, 271-275 (2009).

7. S. Nolte, M. Will, J. Burghoff, and A. Tünnermann, "Femtosecond waveguide writing: a new avenue to three-dimensional integrated optics," Appl. Phys. A 77, 109-111 (2003). 